\title{
ELEMENTAL ABUNDANCES IN THE FAST SOLAR WIND EMANATING FROM CHROMOSPHERIC FUNNELS
}

\author{
Stefano Pucci ${ }^{1}, \varnothing_{\text {ystein Lie-Svendsen }}^{2,4}$, and Ruth Esser ${ }^{3}$ \\ ${ }^{1}$ Institute of Theoretical Astrophysics, University of Oslo, P.O. Box 1029, Blindern, NO-0315 Oslo, Norway; stpucci@yahoo.no \\ ${ }^{2}$ Norwegian Defence Research Establishment, P.O. Box 25, NO-2027 Kjeller, Norway; Oystein.Lie-Svendsen@ffi.no \\ ${ }^{3}$ Institute of Science and Technology, University of Troms $\varnothing$, NO-9037 Troms $\varnothing$, Norway; ruth.esser@uit.no \\ Received 2009 July 8; accepted 2009 December 15; published 2010 January 8
}

\begin{abstract}
We carry out a model study to determine whether a funnel-type flow geometry in the solar wind source region leads to sufficiently fast hydrogen flow to offset heavy element gravitational settling and can thus explain why solar wind abundances are not much smaller than photospheric abundances. We find that high first ionization potential (FIP) elements are more susceptible to gravitational settling than low-FIP elements, which are pulled up by Coulomb drag from protons, and hence the settling is more sensitive to the charge state of the elements than to their mass. Abundances at the top of the chromosphere, and hence solar wind abundances, can change by many orders of magnitude when the funnel areal expansion factor is changed by a small amount. The observed solar wind neon abundance provides the most severe constraint on the expansion, requiring a total flux tube expansion factor of at least 30-40.
\end{abstract}

Key words: solar wind - Sun: abundances - Sun: chromosphere

\section{INTRODUCTION}

Observed solar wind abundances of heavy elements are comparable to photospheric abundances. In particular, abundances in the fast wind originating in polar coronal holes hardly deviate at all from photospheric abundances (von Steiger et al. 2000; Gloeckler \& Geiss 2007). This indicates that the heavier elements are fairly readily pulled out of the chromosphere and into the solar wind. In the chromospheric source region of the solar wind, the hydrogen flow exerts a frictional drag on the heavier elements that counteracts the gravitational pull. However, even for a light element like helium the hydrogen flow in a radially expanding wind, or even a wind from a superradially expanding polar coronal hole, is not fast enough to prevent gravitational settling. Hansteen et al. (1997) found that in order to reproduce the observed solar wind $5 \%$ helium abundance in a radially expanding geometry, the collision frequency for $\mathrm{He}-\mathrm{H}$ collisions in the chromosphere had to be increased by about a factor of 15 above the value obtained from well-established atomic physics. If the correct collision frequency had been used, helium would settle gravitationally in the upper chromosphere and essentially no helium would be present in the solar wind. This gravitational settling problem should be even more severe for elements heavier than helium.

Time-dependent processes in the lower chromosphere might help to keep the material well mixed there. However, above the temperature minimum the chromosphere is convectively stable (e.g., Landau \& Lifshitz 1999) so that some external process must do the mixing. Also, the gravitational settling in the upper chromosphere takes place at timescales of order minutes, e.g., about 5 minutes for Ne near the top of the chromosphere. Although the chromospheric abundance may not be equal to the solar wind abundance (because relative flow speeds may change from the chromosphere to the solar wind), very low abundances at the top of the chromosphere lead to unacceptably low solar wind abundances. The timescale for the mixing process in the upper chromosphere therefore has to be comparable to the settling time, on the order of minutes.

\footnotetext{
4 Also at Institute of Theoretical Astrophysics, University of Oslo, Norway.
}

If, on the other hand, the solar wind originates in funneltype geometries (Dowdy et al. 1986), the flow speed is greatly increased in the chromosphere, resulting in a much larger frictional drag on the minor elements. In this work, we model the flow of minor elements in a chromospheric funnel with a given hydrogen background flow, to study what is required for a funnel to keep solar wind abundances in approximate agreement with observations.

Our model covers only the upper chromosphere and consequently has limitations; the solar wind fluxes of heavy elements, and hence their solar wind abundances, depend on coronal heating of the minor species (Lie-Svendsen \& Esser 2005), which is outside the scope of the model. However, it can be shown analytically (Lie-Svendsen et al. 2003) that due to the gravitational pull on the minor elements there is a maximum flux that can be pulled out of the chromosphere irrespective of the amount of minor ion coronal heating. Preferential heating of minor ions, which is observed in the solar corona (Cranmer et al. 2008), will increase the temperature and flow speed of the ions, but not the ion flux beyond this maximum.

In the upper chromosphere, the ionization degree of hydrogen gradually increases. Because the Coulomb cross section at chromospheric temperatures is of order $10^{3}$ times the neutralneutral and neutral-ion cross sections, low first ionization potential (FIP) elements, which have a high ionization degree in the upper chromosphere, may experience a much stronger frictional drag from hydrogen than do high-FIP elements, even when the $\mathrm{H}$ ionization degree is low.

In the following, we shall calculate this maximum flux for oxygen, and for the high and low FIP elements neon and iron. We choose the latter two elements because these are expected to put the most "severe" constraints on the funnel expansion factor; iron being one of the heaviest elements, and neon being both heavy and a high-FIP element.

\section{THE MODEL}

Our model is based on the picture that some mixing process, not accounted for by the model, maintains photospheric abundances up to a given altitude in the chromosphere. Above this altitude, which is the lower boundary of the model, 


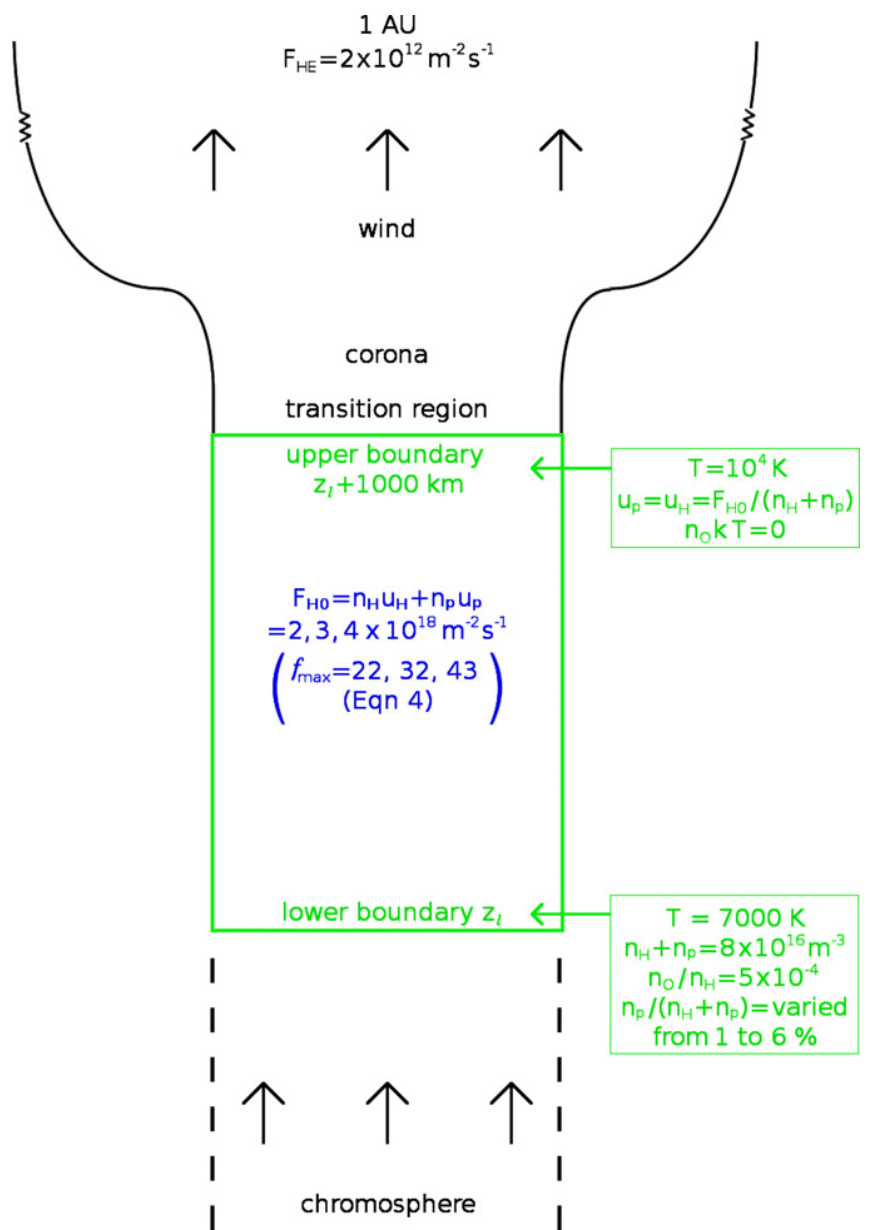

Figure 1. Sketch of the funnel flow geometry assumed. The green-colored area indicates the region covered by the model itself, including the boundary conditions assumed at the lower and upper boundaries. Subscript "O" labels the minor element (exemplified by oxygen). The constant hydrogen flux densities assumed for the three modeled cases are shown in blue.

gravitational settling is allowed to take place. The gravitational settling time can easily be of order months in the midchromosphere, say, so that even very infrequent mixing is sufficient to maintain photospheric abundances in the low chromosphere. In the model domain, we assume that no such mixing, which is inherently a three-dimensional process, occurs during the integration time of the model. We therefore assume horizontal homogeneity and flow only in the vertical direction $z$ (the validity of a one-dimensional treatment is discussed further in Section 4). The equations given below are solved for a $1000 \mathrm{~km}$ thick slab extending from the mid-chromosphere and up to the chromosphere/transition region interface. The funnel is assumed to be vertical with a constant cross section; the funnel expansion taking place above our model in the transition region/corona, in agreement with Dowdy et al. (1986; see also He et al. 2008). Figure 1 sketches the one-dimensional funnel flow geometry, and the section covered by the model.

At chromospheric temperatures we only need to consider neutrals and singly ionized particles. For each species $i$ we solve the continuity equation

$$
\frac{\partial n_{i}}{\partial t}+\frac{\partial\left(n_{i} u_{i}\right)}{\partial z}=n_{j} P_{j i}-n_{i} P_{i j}
$$

where $t$ is time, $n_{i}$ and $u_{i}$ are density and flow speed, and $P_{i j}$ are ionization/recombination rates.
For neutral $\mathrm{H}$ and protons, we solve the momentum equations

$$
\begin{aligned}
& \frac{\partial(n u)_{H(p)}}{\partial t}+\frac{\partial\left(n u^{2}\right)_{H(p)}}{\partial z} \\
& =-\frac{a}{m_{p}} \frac{\partial\left(n_{H(p)} k T\right)}{\partial z}-g n_{H(p)}+n_{p(H)} u_{p(H)} P_{\mathrm{pH}(\mathrm{Hp})} \\
& \quad-n_{H(p)} u_{H(p)} P_{\mathrm{Hp}(\mathrm{pH})}+k_{\mathrm{mt}} n_{H(p)} n_{p(H)}\left(u_{p(H)}-u_{H(p)}\right),
\end{aligned}
$$

where $m_{p}, k$, and $T(z)$ are the proton mass, Boltzmann's constant, and temperature (equal for all species), respectively; $g$ is the constant gravitational acceleration; and $a=1$ for $\mathrm{H}$ and $a=2$ for protons (accounting for the electric field contribution). For the friction terms from elastic collisions we adopt the rate coefficient $k_{\mathrm{mt}}(T)=1.9 \times 10^{-14} \sqrt{T / 10^{4} \mathrm{~K}} \mathrm{~m}^{3} \mathrm{~s}^{-1}$, in good agreement with the value by Schultz et al. (2008) at chromospheric temperatures. For $P_{\mathrm{pH}}$ we use the radiative recombination rates given by Arnaud \& Rothenflug (1985), which agree with more recent calculations (Péquignot et al. 1991; Verner \& Ferland 1996) at $T \lesssim 10^{6} \mathrm{~K}$, while for simplicity we choose a constant photoionization rate $P_{\mathrm{Hp}}$ (to be specified below). At chromospheric temperatures direct ionization (collisions with electrons) is negligible by comparison.

For the minor elements, the momentum equation reads

$$
\begin{aligned}
\frac{\partial\left(n_{i} u_{i}\right)}{\partial t}+\frac{\partial\left(n_{i} u_{i}^{2}\right)}{\partial z}= & -\frac{1}{m_{i}} \frac{\partial\left(n_{i} k T\right)}{\partial z}-\frac{n_{i} Z_{i}}{m_{i}} \frac{1}{n_{p}} \frac{\partial\left(n_{p} k T\right)}{\partial z}-g n_{i} \\
& +n_{j} u_{j} P_{j i}-n_{i} u_{i} P_{i j}+\sum_{l=H, p} n_{i} v_{i l}\left(u_{l}-u_{i}\right),
\end{aligned}
$$

where $Z_{i}$ is the particle charge in units of the elementary charge. For ionization we use the direct rates of Arnaud \& Rothenflug (1985), except iron (Fe), which we take from Arnaud $\&$ Raymond (1992). Their rate is also used for Fe dielectronic recombination. Radiative recombination rates, and dielectronic recombination rates for elements other than iron, are obtained from Shull \& van Steenberg (1982). To bring the ionization of Fe up to values given by Vernazza et al. (1981), i.e., almost fully ionized, an Fe photoionization rate of $10^{-2} \mathrm{~s}^{-1}$ is added. At chromospheric temperatures the chosen recombination rates agree with the more recent compilation of Mazzotta et al. (1998), except that our rate for dielectronic recombination of neon is significantly smaller. However, for neon radiative recombination dominates and the difference in the total recombination rate is negligible.

The collision frequencies $v_{\mathrm{il}}$ in Equation (3), corresponding to neutral-neutral, neutral-ion, and Coulomb collisions are taken from Schunk (1977). For oxygen (O), resonant charge transfer with $\mathrm{H}$ is a dominant process, determining the $\mathrm{O}$ ionization degree and adding a large term to the frictional drag from H. Denoting O I(O II) by subscript $1(2)$, the rates are $P_{12(21)}=n_{p(H)} C_{I(R)}$. At chromospheric temperatures the rate coefficients are $C_{I} \simeq 0.91 \times 10^{-15} \mathrm{~m}^{3} \mathrm{~s}^{-1}$ and $C_{R} \simeq$ $10^{-15} \mathrm{~m}^{3} \mathrm{~s}^{-1}$ (Arnaud \& Rothenflug 1985). In ionization equilibrium, the $\mathrm{O}$ and $\mathrm{H}$ ionization degrees are therefore almost equal, $n_{2} / n_{1} \approx P_{12} / P_{21} \approx 0.91 n_{p} / n_{H}$.

Because of the strong collisional coupling between species in the chromosphere and the weak temperature gradient we assume that all species have a common temperature $T(z)$. We do not solve the energy equation. Instead we prescribe a temperature 


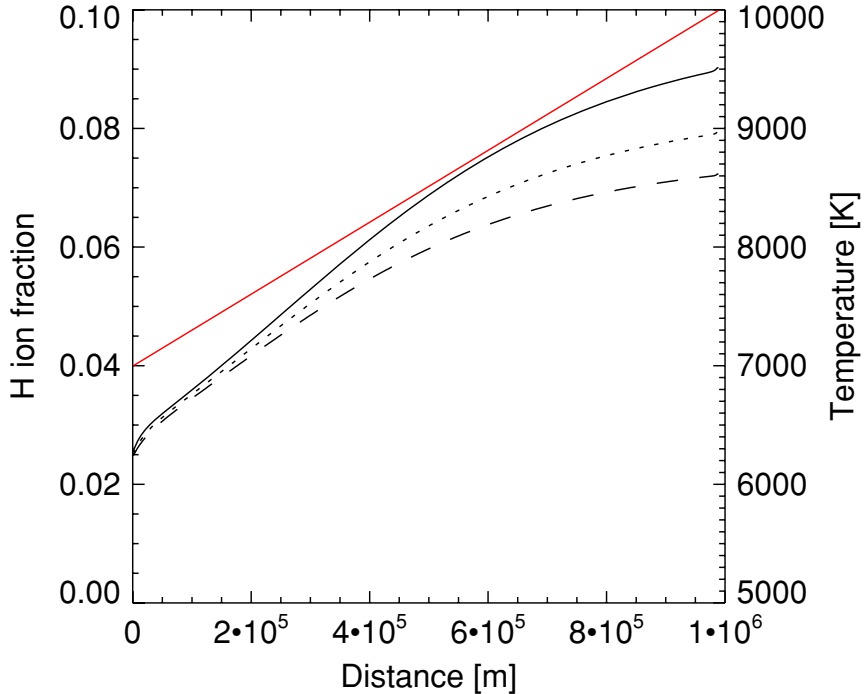

Figure 2. Hydrogen ion fraction $n_{p} /\left(n_{\mathrm{H}}+n_{p}\right)$ for $f_{\max }=22$ (solid), 32 (dotted), and 43 (dashed curve), and the adopted temperature profile (red line).

profile, increasing linearly from $7000 \mathrm{~K}$ at the lower boundary of the slab $\left(z=z_{l}\right)$ to $10^{4} \mathrm{~K}$ at the upper boundary $\left(z=z_{u}\right)$ (also shown in Figure 2). The hydrogen density at the lower boundary is set to $n_{H}+n_{p}=8 \times 10^{16} \mathrm{~m}^{-3}$. The material streaming in through the lower boundary is assumed to have an ionization degree close to the ionization equilibrium value $n_{p} / n_{\mathrm{H}}=P_{\mathrm{H} p} / P_{p \mathrm{H}}$, and hence varies depending on the chosen $P_{\mathrm{H} p}$. The hydrogen density at the lower boundary agrees with model C of Vernazza et al. (1981) at the same temperature, although that was a model of the average quiet Sun with no outflow and therefore not directly comparable to our funnel model.

Similar to the minor elements, the solar wind mass flux depends on the coronal energy balance and cannot be determined by a model spanning only the chromosphere. Hence, the $\mathrm{H}$ flux density, $F_{\mathrm{H}} \equiv n_{H} u_{H}+n_{p} u_{p}$, a constant in steady state, must be provided as an input to the model. At the upper boundary we set $u_{p}=u_{H}=F_{H 0} /\left(n_{H}\left(z_{u}\right)+n_{p}\left(z_{u}\right)\right)$, where $F_{H 0}$ has a fixed prescribed value. When the model is integrated to steady state $F_{\mathrm{H}}=F_{\mathrm{H} 0}$ throughout the slab. $F_{\mathrm{H} 0}$ is the essential model input parameter. In a steady-state solar wind, the particle flux is constant and hence $F_{\mathrm{H} 0}$ can be related to the funnel expansion factor $f_{\max }$, the increase in flow tube area from the chromosphere to infinity relative to a radially expanding flow tube,

$$
F_{\mathrm{H} 0}=f_{\max }\left(\frac{1 \mathrm{AU}}{1 R_{\odot}}\right)^{2} F_{\mathrm{HE}},
$$

where $F_{\mathrm{HE}} \approx 2 \times 10^{12} \mathrm{~m}^{-2} \mathrm{~s}^{-1}$ is the observed fast solar wind proton flux density at Earth orbit. At the lower boundary the proton and neutral hydrogen velocities are set equal, and the value is allowed to float, using the scheme of Korevaar \& van Leer (1988), so that in the steady state $u_{\mathrm{H}}\left(z_{l}\right)=u_{p}\left(z_{l}\right)=$ $F_{\mathrm{H} 0} /\left(n_{\mathrm{H}}\left(z_{l}\right)+n_{p}\left(z_{l}\right)\right)$. Hydrogen is assumed to be in ionization equilibrium at the lower boundary.

At the start of the $\mathrm{H}$ time integration, neutral $\mathrm{H}$ is in hydrostatic equilibrium and the ionization degree is set to $10^{-2}$ everywhere. The model is integrated with a semi-implicit time integration scheme, typically for $t=2 \times 10^{4} \mathrm{~s}$ which is ample time for a steady state with constant $F_{\mathrm{H}}$ to be reached.

The minor ion equations are solved with the steady-state $\mathrm{H}$ solution as a fixed background. The particles of element $i$
Table 1

Model Boundary Conditions

\begin{tabular}{|c|c|}
\hline Model Parameter & Boundary Condition \\
\hline \multicolumn{2}{|c|}{ Lower Boundary $\left(z=z_{l}\right)$} \\
\hline H density & $n_{\mathrm{H}}=8 \times 10^{16} \mathrm{~m}^{-3}$ \\
\hline $\mathrm{H}$ ion fraction & $n_{p} /\left(n_{\mathrm{H}}+n_{p}\right) \approx P_{\mathrm{H} p} /\left(P_{\mathrm{H} p}+P_{p \mathrm{H}}\right)$ \\
\hline Minor element abundance & $A_{i}^{0}=5 \times 10^{-4}$ \\
\hline Minor ion fraction & $n_{2} /\left(n_{1}+n_{2}\right)=P_{12} /\left(P_{12}+P_{21}\right)$ \\
\hline \multicolumn{2}{|c|}{ Upper Boundary $\left(z=z_{u}=z_{l}+10^{6} \mathrm{~m}\right)$} \\
\hline H flux density & $F_{\mathrm{H} 0}$ \\
\hline Minor element pressure & $n_{i} k T=0$ \\
\hline
\end{tabular}

streaming in through the lower boundary are assumed to have an ionization degree corresponding to ionization equilibrium at that temperature, and a fixed absolute abundance (i.e., relative to $\mathrm{H}$ ) which is close to the observed $\mathrm{O}$ abundance, $A_{i}^{0} \equiv n_{i}^{0} / n_{\mathrm{H}}^{0}=5 \times 10^{-4}$ (choosing the same abundance for all elements facilitates comparison).

The maximum flux of the minor element, which is the main model result, is obtained by setting the minor element pressure to zero at the upper boundary. The minor element flow speed is not fixed, neither at the lower nor the upper boundary, but allowed to adjust itself to satisfy local force balance by solving the momentum equation also at the boundaries. In the hypothetical case of no hydrogen flow and the minor element initially at rest in hydrostatic equilibrium, the zero pressure assumption at the upper boundary would cause a sound wave to propagate downward and the resulting reduced pressure would then induce upflow through the lower boundary. With nonzero hydrogen flow, the steady-state minor element flow speed at the lower boundary is influenced both by $F_{\mathrm{H}}$ and the minor element pressure assumed at the upper boundary.

The boundary conditions used are summarized in Table 1 and also indicated in Figure 1.

At the start of the calculation, the neutral minor species is assumed to have the same density scale height as $\mathrm{H}$, while the ion fraction is constant corresponding to ionization equilibrium at the lower boundary. The minor species is thus far from hydrostatic equilibrium initially and, depending on the minor species degree of ionization and the magnitude of the $\mathrm{H}$ flux density $F_{\mathrm{H}}$, it will undergo gravitational settling. Reaching a true steady state, in which the minor species particle flux density is also constant with altitude, may therefore take a long time, particularly in cases with extensive gravitational settling. Hence, the minor species transport equations are integrated forward in time much longer than $\mathrm{H}$, typically for $t=2 \times 10^{5} \mathrm{~s}$.

\section{RESULTS}

We consider three values for the input $\mathrm{H}$ flux, $F_{\mathrm{H} 0}=2,3$, and $4 \times 10^{18} \mathrm{~m}^{-2} \mathrm{~s}^{-1}$, corresponding to $f_{\max } \approx 22,32$, and 43 (from Equation (4)). These values have been chosen because they turn out to cover the "interesting" parameter region: with smaller values of $f_{\max }$ we essentially get severe settling of all elements (as mentioned in Section 1, $f_{\max }=1$ leads to settling even for such a light element as helium), while larger values of $f_{\text {max }}$ lead to almost no settling.

We first choose a hydrogen photoionization rate $P_{\mathrm{Hp}} \approx$ $4 \times 10^{-5} \mathrm{~s}^{-1}$. The resulting hydrogen ionization degrees are shown in Figure 2, showing that it increases gradually from $3 \%$ at the lower boundary to almost $10 \%$ at the upper boundary. The ionization degree at the upper boundary assuming ionization 

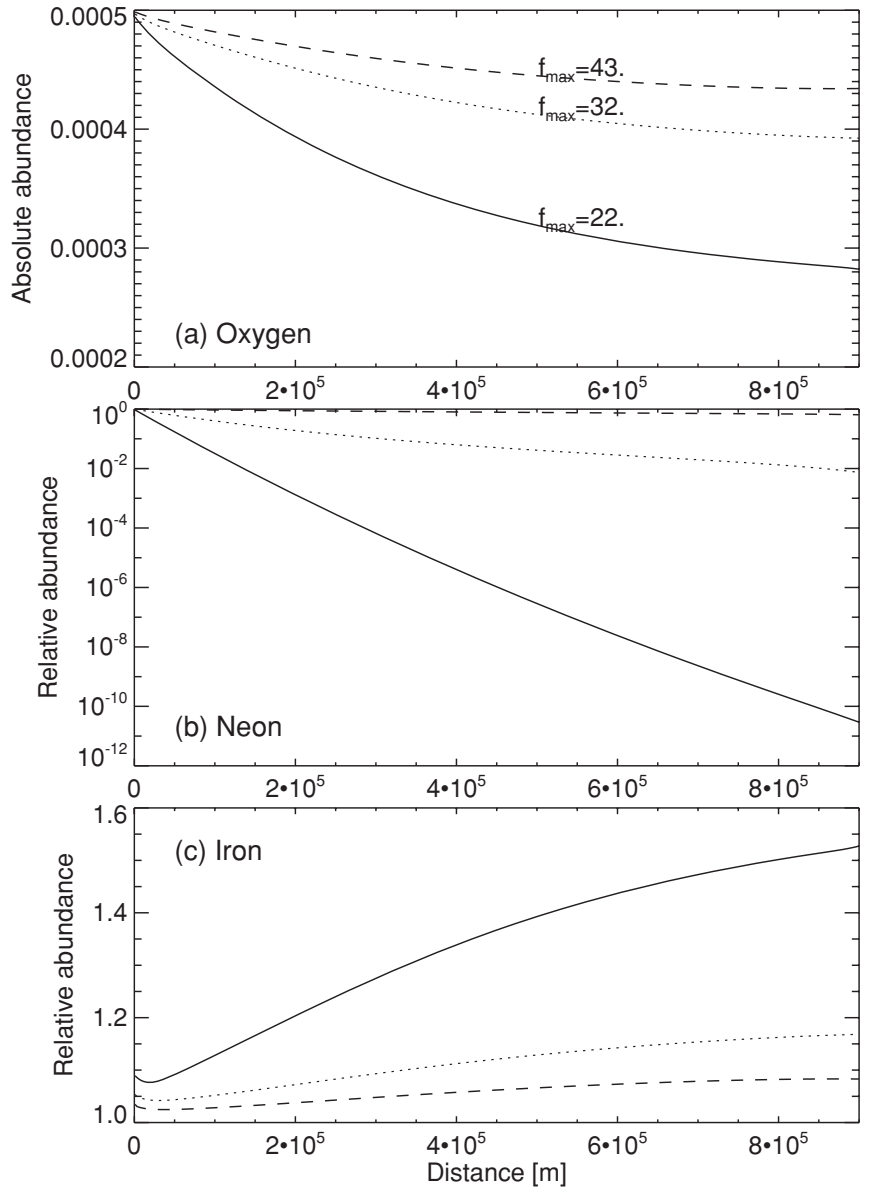

Figure 3. (a) Steady-state oxygen abundance, $n_{\mathrm{O}} / n_{\mathrm{H}}$, for the three background solutions corresponding to $f_{\max }=22,32$, and 43; (b) Ne abundance; and (c) $\mathrm{Fe}$ abundance, both relative to $\mathrm{O}$ for the same $\mathrm{H}$ models.

equilibrium would have been about $40 \%$. Hence, the rapid flow in all three funnels brings hydrogen far from ionization equilibrium.

\subsection{Oxygen}

Figure 3 (upper panel) shows the $\mathrm{O}$ abundances obtained with these background $\mathrm{H}$ solutions. We note that the larger the $\mathrm{H}$ flux, the less the $\mathrm{O}$ abundance decreases through the layer. In the $f_{\max }=43$ case, the $\mathrm{O}$ absolute abundance does not decrease by more than about $10 \%$ through the layer, showing that the frictional drag from $\mathrm{H}$ is sufficient to prevent gravitational settling of $\mathrm{O}$. In the $f_{\max }=22$ case, the settling is more pronounced, with the abundance decreasing by more than $40 \%$ through the layer.

This is in stark contrast to hydrostatic equilibrium, in which case neutral $\mathrm{O}$ has a density scale height $1 / 16$ that of $\mathrm{H}$ and the abundance at the top would be only of order $10^{-27}$ times the abundance at the bottom of the slab. Hence the presence of $\mathrm{H}$ flow, although highly subsonic, has a dramatic impact on the $\mathrm{O}$ abundance.

The abundance at the top of the slab is not equal to the solar wind abundance because the minor species do not in general flow at the $\mathrm{H}$ flow speed in the upper chromosphere. Changes in relative flow speed between $\mathrm{O}$ and $\mathrm{H}$ between the top of the chromosphere and the solar wind will cause changes in the $\mathrm{O}$ abundance. In a steady-state flow, flux conservation implies that the solar wind $\mathrm{O}$ abundance, $A_{\mathrm{O}}^{\mathrm{sw}}$, is related to the (constant)
Table 2

Maximum Fractionation, Defined in Equation (6), for the Three Chosen Funnel Expansion Factors $f_{\max }$ and $P_{\mathrm{Hp}}=4 \times 10^{-5} \mathrm{~s}^{-1}$

\begin{tabular}{lccc}
\hline \hline \multicolumn{1}{c}{$f_{\max }=$} & 22 & 32 & 43 \\
\hline$f[\mathrm{O}]$ & 0.30 & 0.53 & 0.64 \\
$f[\mathrm{Ne}] / f[\mathrm{O}]$ & $4.2 \times 10^{-12}$ & $1.7 \times 10^{-3}$ & 0.25 \\
$f[\mathrm{Fe}] / f[\mathrm{O}]$ & 2.5 & 1.6 & 1.4 \\
\hline
\end{tabular}

flux densities in the chromosphere,

$$
A_{\mathrm{O}}^{\mathrm{sw}} \equiv \frac{n_{\mathrm{O}}^{\mathrm{sw}}}{n_{p}^{\mathrm{sw}}}=\frac{F_{\mathrm{O}}}{F_{\mathrm{H}}} \frac{u_{\mathrm{H}}^{\mathrm{sw}}}{u_{\mathrm{O}}^{\mathrm{sw}}},
$$

where superscript "sw" denotes solar wind densities and flow speeds (averaged over charge states), and $F_{\mathrm{O}}$ is the $\mathrm{O}$ chromospheric particle flux density $\left(F_{\mathrm{O}}=n_{1} u_{1}+n_{2} u_{2}\right.$ where subscripts 1 and 2 denote neutral and ionized components). In the solar wind far from the Sun the minor ions flow at speeds that do not deviate from the proton speed by more than the local Alfvén speed (e.g., von Steiger et al. 1995), which is about 5\% of the flow speed at $1 \mathrm{AU}$. Hence, $u_{\mathrm{O}}^{\mathrm{sw}} \approx u_{\mathrm{H}}^{\mathrm{sw}}$. We define the $\mathrm{O}$ fractionation, $f[\mathrm{O}]$, as the ratio of the solar wind abundance to the abundance at the lower boundary of the model (the "photosphere"). We then have from (5)

$$
f[\mathrm{O}] \equiv \frac{\frac{n_{\mathrm{O}}^{\mathrm{sw}}}{n_{p}^{\mathrm{sw}}}}{\frac{n_{\mathrm{O}}^{0}}{n_{\mathrm{H}}^{0}}}=\frac{n_{\mathrm{H}}^{0}}{n_{\mathrm{O}}^{0}} \frac{F_{\mathrm{O}}}{F_{\mathrm{H}}} \frac{u_{\mathrm{H}}^{\mathrm{sw}}}{u_{\mathrm{O}}^{\mathrm{sw}}} \approx \frac{n_{\mathrm{H}}^{0}}{n_{\mathrm{O}}^{0}} \frac{F_{\mathrm{O}}}{F_{\mathrm{H}}}=\frac{u_{\mathrm{O}}^{0}}{u_{\mathrm{H}}^{0}},
$$

where superscript " 0 " denotes total densities (summed over charge states) and flow speeds (averaged over charge states) evaluated at the lower boundary. Hence, to a good approximation, the solar wind fractionation is just the ratio of the minor element to $\mathrm{H}$ flow speed at the lower boundary.

For the three $\mathrm{H}$ solutions with $f_{\max }=22,32$, and 43 we find $f[\mathrm{O}]=0.30,0.53$, and 0.64 (summarized in Table 2), showing that $\mathrm{O}$ is depleted relative to the "photosphere" (the lower boundary) by a factor of 2-3, and least in the most narrow funnel (largest $f_{\max }$ ). Again we emphasize that these are the largest possible values of $f[\mathrm{O}]$ for the chosen flow geometries; if the coronal heating is insufficient to heat the $\mathrm{O}$ streaming into the corona, the values will be reduced.

\subsection{Neon}

While $\mathrm{O}$ shows modest variation in the maximum solar wind fractionation for the chosen values of $f_{\max }$, the Ne fractionation is extremely sensitive to the value of $f_{\max }$, as shown in Table 2. For $f_{\max }=22$ and 32 essentially no Ne can be pulled out of the chromosphere, while for $f_{\max }=43 \mathrm{Ne}$ is "only" depleted by a factor of 4 relative to $\mathrm{O}$.

Figure 3 (middle panel) shows the $\mathrm{Ne}$ abundance relative to O. In the $f_{\max }=43$ case, the neon abundance remains close to the oxygen abundance throughout the slab (recall that we have assumed that all minor elements have the same abundance at the lower boundary). In the $f_{\max }=32$ case, the abundance near the upper boundary is reduced by more than a factor of 100 compared with the $f_{\max }=43$ case, while in the $f_{\max }=22$ case the abundance decreases enormously through the slab showing that essentially no neon remains at the top.

Hence, there is a quite sudden transition for $\mathrm{Ne}$ around $f_{\max } \approx 30-40$ : for larger values of $f_{\max } \mathrm{Ne}$ is pulled out of the chromosphere, and although there will be a depletion it is rather 


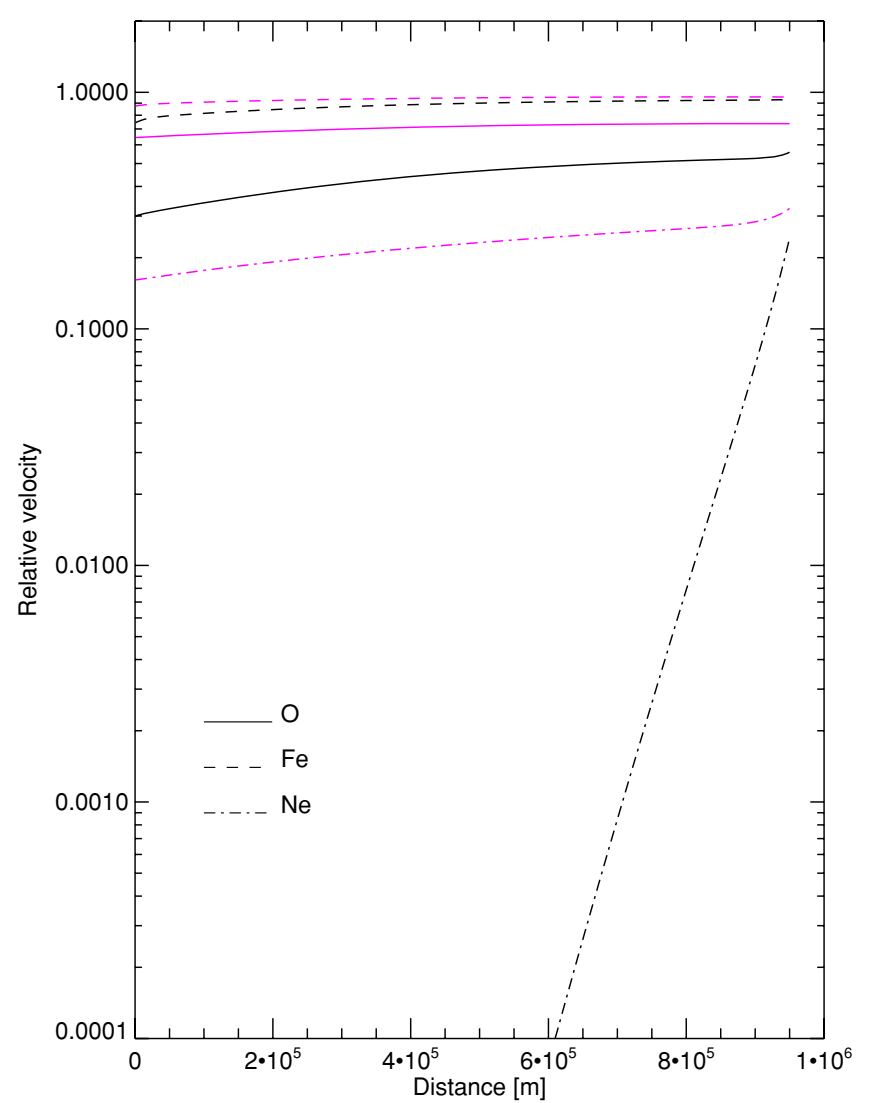

Figure 4. Flow speeds relative to $\mathrm{H}$ for $\mathrm{O}, \mathrm{Fe}$, and $\mathrm{Ne}$ for $f_{\max }=22$ (black curves) and $f_{\max }=43$ (purple curves). The relative flow speed is defined as $u_{\text {rel }}=\frac{n_{1} u_{1}+n_{2} u_{2}}{n_{1}+n_{2}} \frac{n_{\mathrm{H}}+n_{p}}{n_{\mathrm{H}} u_{\mathrm{H}}+n_{p} u_{p}}$, where 1 and 2 indicate the neutral and ionized components of the minor element, respectively.

small and can certainly be in agreement with in situ observations. For smaller $f_{\max }$, gravitational settling "suddenly" dominates, and no $\mathrm{Ne}$ emanates from the top of the chromosphere into the corona. And, again, these are the maximum solar wind abundances that can be obtained, irrespective of the coronal heating of minor ions.

The main reason why Ne undergoes such extensive gravitational settling, while $\mathrm{O}$ does not, is because $\mathrm{Ne}$ is not subject to resonant charge exchange with $\mathrm{H}$ (the FIP of $\mathrm{Ne}$ is $21.6 \mathrm{eV}$ ), and that $\mathrm{Ne}$ is mostly neutral in the chromosphere (the ionization degree at the lower boundary of the model is less than $10^{-11}$ ). Hence, the frictional drag from $\mathrm{H}$ will be smaller than for $\mathrm{O}$. Had we used values for $f_{\max }$ much smaller, also $\mathrm{O}$ would have undergone gravitational settling.

The very different behavior of $\mathrm{Ne}$ and $\mathrm{O}$ is also illustrated in the flow speeds of Figure 4. For $f_{\max }=22$, Ne is essentially at rest, decoupled from the $\mathrm{H}$ flow, but even in the more rapid $\mathrm{H}$ flow of the $f_{\max }=43$ case the Ne flow speed is much lower than the $\mathrm{H}$ speed $\left(u_{p} \approx u_{\mathrm{H}}\right.$ in all solutions). Oxygen also flows slower than $\mathrm{H}$, but the relative difference is much smaller.

\subsection{Iron}

Table 2 shows that iron is readily pulled out of the chromosphere for all three values of $f_{\max }$, with a maximum solar wind fractionation (from Equation (6)) that is even larger than that of oxygen. Figure 3 (lower panel) shows the Fe abundance relative to $\mathrm{O}$. We note that the relative abundance even increases through the slab. The increase in relative abundance and solar

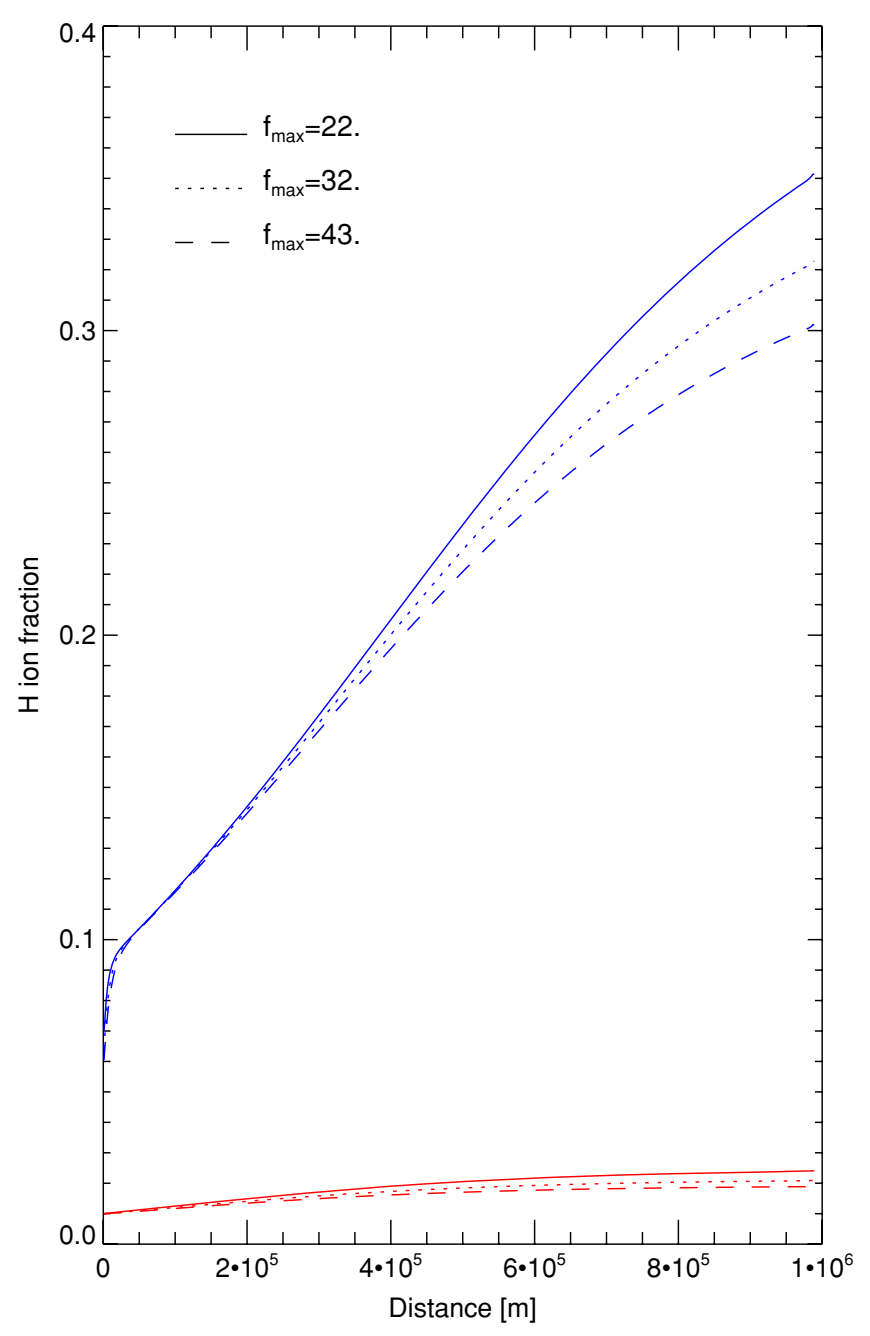

Figure 5. $\mathrm{H}$ ion fraction for $P_{\mathrm{Hp}}=5 \times 10^{-6} \mathrm{~s}^{-1}$ (red curves) and $P_{\mathrm{Hp}}=$ $4 \times 10^{-4} \mathrm{~s}^{-1}$ (blue curves) and for the different funnel expansion factors $f_{\max }$.

wind fractionation is largest for the smallest $f_{\max }$, which merely reflects that the $\mathrm{O}$ abundance decreases more for smaller $f_{\max }$.

Figure 4 also illustrates that iron does not settle gravitationally, with a flow speed that remains close to the hydrogen flow speed in all the chosen flow geometries. Fe is pulled out of the chromosphere because it is ionized. Although $\mathrm{H}$ is mostly neutral in these models, the few protons present provide sufficient drag to prevent $\mathrm{Fe}$ ions from settling since the Coulomb cross section at chromospheric temperatures is of order $10^{3}$ larger than the ion-neutral cross section.

\subsection{Varying the Hydrogen Ionization Degree}

The above results were obtained assuming a hydrogen photoionization rate $P_{\mathrm{Hp}} \approx 4 \times 10^{-5} \mathrm{~s}^{-1}$. We now consider a much lower rate, $P_{\mathrm{Hp}}=5 \times 10^{-6} \mathrm{~s}^{-1}$, and a much higher rate, $P_{\mathrm{Hp}}=$ $4 \times 10^{-4} \mathrm{~s}^{-1}$. The resulting $\mathrm{H}$ ionization degree in the different flow geometries is shown in Figure 5, showing that we now have ionization degrees varying from $1 \%$ to $2 \%$ up to more than $30 \%$. Since we do not solve the energy equation, we can in principle obtain an arbitrary ionization degree with the chosen temperature profile. At very high $\mathrm{H}$ ionization one may question whether a chromosphere may still exist, since the radiative cooling from Ly $\alpha$ radiation then becomes less efficient. The highest ionization rates in Figure 5 may therefore be more appropriate for the 
Table 3

Maximum Fractionation, Defined in Equation (6), for Low and High $\mathrm{H}$ Photoionization Rates

\begin{tabular}{lccc}
\hline \hline \multicolumn{1}{c}{$f_{\max }=$} & 22 & 32 & 43 \\
\hline \multicolumn{4}{c}{$P_{\mathrm{Hp}}=5 \times 10^{-6} \mathrm{~s}^{-1}$} \\
\hline$f[\mathrm{O}]$ & 0.23 & 0.48 & 0.61 \\
$f[\mathrm{Ne}] / f[\mathrm{O}]$ & $8.8 \times 10^{-12}$ & $2.0 \times 10^{-3}$ & 0.26 \\
$f[\mathrm{Fe}] / f[\mathrm{O}]$ & 2.6 & 1.5 & 1.3 \\
\hline \multicolumn{5}{c}{$\mathrm{P}_{\mathrm{Hp}}=4 \times 10^{-4} \mathrm{~s}^{-1}$} \\
\hline$f[\mathrm{O}]$ & 0.47 & 0.63 & \\
$f[\mathrm{Ne}] / f[\mathrm{O}]$ & $1.3 \times 10^{-12}$ & $1.0 \times 10^{-3}$ & 0.71 \\
$f[\mathrm{Fe}] / f[\mathrm{O}]$ & 1.3 & 1.2 & 1.2 \\
\hline
\end{tabular}

low transition region where the temperature gradient is much larger than we have assumed. However, the point here is simply to investigate whether our results are sensitive to the ionization degree of the upper chromosphere, and we therefore choose a high ionization degree as the upper limit.

It turns out that the results are not very sensitive to the chromospheric ionization, as shown in Table 3. Despite a factor of 20-30 increase in $\mathrm{H}$ ionization between the two cases, the $\mathrm{O}$ (absolute) fractionation has only increased by a factor of 2 in the $f_{\max }=22$ case, and even less for larger funnel expansions. The $\mathrm{Ne}$ and $\mathrm{Fe}$ relative (to $\mathrm{O}$ ) fractionations actually decrease when the $\mathrm{H}$ ionization degree increases. For neon, this is mostly due to the neon-proton collisional cross section being somewhat smaller than the neon-neutral $\mathrm{H}$ cross section. For iron, which is ionized in all cases, the flux is almost independent of the hydrogen ionization (showing that it remains closely coupled to the $\mathrm{H}$ flow), so that the decrease in relative $\mathrm{Fe}$ fractionation is caused almost entirely by the increase in the $\mathrm{O}$ flux.

Apart from these small changes, we may conclude that our main result is to a large extent independent of the ionization degree of the chromosphere: with the chosen funnel expansion factors, both oxygen and iron are pulled out of the chromosphere into the corona. Assuming that the coronal minor ion heating is sufficiently large to support this incoming flux, the solar wind abundances will then be of the same order of magnitude as the photospheric abundances. Neon on the other hand is not pulled out at all for $f_{\max }=22$, while it is pulled out with $f_{\max }=43$; hence, for neon there is an abrupt transition at expansion factors of about 30-40.

\section{DISCUSSION}

We have shown that the maximum flux of minor elements pulled out of the chromosphere by frictional drag from $\mathrm{H}$ can be extremely sensitive to the H flux density, or, equivalently, to the funnel expansion factor $f_{\max }$. The essential behavior can be demonstrated analytically making some simplifying assumptions: the temperature is assumed to be constant throughout the slab; $\mathrm{H}$ is neutral and in a steady-state flow with a density falling off with the constant scale height $h=k T /\left(m_{p} g\right)$; the minor element degree of ionization does not change through the slab; ionization, recombination, electric field, and inertial terms are all neglected in the respective equations of the minor element. In this case, the time independent version of Equations (1) and (3) can be integrated analytically and the constant minor element particle flux density obtained as a function of the minor element densities at the lower and upper boundaries. Furthermore, by setting the minor element density to zero at the upper boundary, the maximum flux density (which, as remarked, is the only parameter we can obtain without also including the corona and solar wind in the model) is obtained (Lie-Svendsen et al. 2003):

$$
\max \left(F_{i}\right)=A_{i}^{0} \frac{g}{\hat{v}} \frac{m_{p}}{m_{i}} \frac{\gamma}{e^{\gamma \tau}-1},
$$

where (again) $A_{i}^{0} \equiv n_{i}^{0} / n_{\mathrm{H}}^{0}$ is the absolute abundance at the lower boundary, $\hat{v} \equiv v_{i \mathrm{H}} / n_{\mathrm{H}}$, and

$$
\begin{gathered}
\gamma \equiv \frac{m_{i}}{m_{p}}\left(1-\frac{\hat{v} F_{\mathrm{H}}}{g}\right)-1, \\
\tau \equiv \frac{m_{p} g\left(z_{u}-z_{l}\right)}{k T} .
\end{gathered}
$$

Here, $v_{i \mathrm{H}}$ is the "effective" collision frequency (taking into account that the minor element may be partially ionized) and $\hat{v}$ is a constant when the temperature is constant and the minor element ionization degree does not vary. $\tau$ is the slab thickness in units of the $\mathrm{H}$ scale height; the chosen slab thickness of the numerical model, $z_{u}-z_{l}=10^{6} \mathrm{~m}$, corresponds to $\tau \approx 5$ at 7000 K. From Equations (6) and (7) the maximum possible absolute fractionation of element $i$ is also obtained,

$$
\max (f[\mathrm{i}])=\frac{\frac{g}{\hat{v} F_{\mathrm{H}}}\left(1-\frac{m_{p}}{m_{i}}\right)-1}{e^{\gamma \tau}-1} .
$$

Since $m_{i} \gg m_{p}$ (except helium), $\gamma \gg 1$ when $F_{\mathrm{H}}<g / \hat{v}$ and $\gamma \ll-1$ when $F_{\mathrm{H}}>g / \hat{v}$. Unless the slab is very thin $(\tau \ll 1)$, and except the singular case when the $\mathrm{H}$ flux density is almost equal to $g / \hat{v}$, the system will be in one of two very different regimes:

$$
\begin{gathered}
\max (f[\mathrm{i}]) \approx\left[\frac{g}{\hat{v} F_{\mathrm{H}}}\left(1-\frac{m_{p}}{m_{i}}\right)-1\right] e^{-\gamma \tau} \quad \text { if } F_{\mathrm{H}}<\frac{g}{\hat{v}}, \\
\max (f[\mathrm{i}]) \approx 1-\frac{g}{\hat{v} F_{\mathrm{H}}}\left(1-\frac{m_{p}}{m_{i}}\right) \quad \text { if } F_{\mathrm{H}}>\frac{g}{\hat{v}} .
\end{gathered}
$$

In the limiting case $F_{\mathrm{H}} \ll g / \hat{v}$ we have for Ne $\gamma \tau \approx 100$, and hence $\max (f[\mathrm{Ne}])=0$ from Equation (11). This explains why we obtained the vanishing small $\mathrm{Ne}$ fractionation in the $f_{\max }=22$ case. In the opposite extreme with a very large $\mathrm{H}$ flux (narrow funnel), $F_{\mathrm{H}} \gg g / \hat{v},(12)$ yields

$$
\lim _{F_{\mathrm{H}} \gg g / \hat{v}} \max (f[\mathrm{i}])=1 .
$$

Provided that the minor ion coronal heating is sufficiently strong, a very narrow funnel thus yields no fractionation at all, $f[\mathrm{i}]=1$. (If the coronal heating is not sufficient, the zero pressure assumption at the top of the chromosphere is no longer appropriate and the flux is reduced from this maximum value.) The "sudden" transition between expressions (11) and (12) at $F_{\mathrm{H}}=g / \hat{v}$ also explains why we got the enormous rise in $\mathrm{Ne}$ fractionation when $f_{\max }$ was increased modestly from 22 to 32 , and further to $f_{\max }=43$.

Whether a minor species is pulled out of the chromosphere (thus having a nonvanishing solar wind fractionation) depends critically on the charge state of the minor species in the chromosphere. Despite that Fe is nearly 3 times heavier than 
$\mathrm{Ne}$, ionized $\mathrm{Fe}$ is pulled out of a funnel with $f_{\max }=22$ while neutral $\mathrm{Ne}$ is not, as shown in Figure 3. Increased ionization increases the importance of Coulomb collisions, leading to an increase in $\hat{v}$ and hence to a transition from the $F_{\mathrm{H}}<g / \hat{v}$ to the $F_{\mathrm{H}}>g / \hat{v}$ regime. For minor elements with a very low ionization degree in the chromosphere, so that neutral-H and neutral-proton collisions dominate, $\hat{v} \propto 1 / m_{i}$, and one would expect that heavier elements should be more susceptible to gravitational settling than lighter elements. However, $\hat{v}$ also depends on the collision cross section and thus the "size" of the neutral minor atom, and hence no general mass dependence of the gravitational settling can be inferred; for instance, the neutral neon and neutral iron collision frequencies are actually quite similar.

Our focus has been on the requirements necessary for heavy elements, particularly high-FIP elements like neon, to be pulled out of the chromosphere at all. However, because the ionization degree of the heavy element is so critical, we do find that the solar wind abundance of the low-FIP element iron, relative to oxygen, becomes higher than the photospheric relative abundance, and that the effect is largest for smaller funnel expansion factors (when oxygen becomes more depleted in the solar wind), while the high-FIP element neon definitely becomes depleted even when the expansion factor is large. Thus, the competition between frictional drag from hydrogen and gravitational settling can explain the relative FIP fractionation observed in the solar wind, confirming that the upper chromosphere, where lowFIP elements become ionized, may be the seat of this effect. However, the frictional drag can only lead to an abundance enhancement relative to oxygen in the solar wind, it cannot lead to an absolute abundance enhancement relative to hydrogen. Because the frictional pull, needed to prevent gravitational settling, requires that the minor element flows more slowly than hydrogen in the chromosphere, Equation (6) shows that the absolute fractionation will always be less than unity; in other words, the heavy elements must always have a solar wind absolute abundance that is smaller than (or nearly equal to) the "photospheric" abundance. An absolute enrichment of low-FIP elements like iron requires that they are already enriched at the lower boundary of the model.

The ionization of hydrogen and minor elements may thus cause an increase in the abundance relative to oxygen, but never in the absolute abundance. It has previously been suggested that a rapid ionization process may lead to absolute FIP fractionation (Peter 1998). However, that study only considered what happens in the ionization layer, disregarding that the minor elements may already be depleted by the time they reach the lower boundary of the ionization layer, and necessarily must flow more slowly than hydrogen just below the layer. The ionization process may then lead to a temporary increase in the minor element flow velocity, accompanied by a corresponding decrease in absolute abundance, but will not affect the solar wind abundances. This flaw has already been pointed out by McKenzie et al. (1998) and McKenzie (2000), who did not account for gravity, however, and therefore claimed that the chromosphere cannot have any impact on solar wind absolute abundances. With gravity included, the chromospheric flow may indeed affect solar wind absolute abundances, but the effect must always be negative; absolute solar wind abundances are always reduced relative to photospheric abundances, but the faster the flow (more narrow funnel) the smaller is the reduction.

The most recent re-analysis of SWICS/Ulysses in situ data shows that there is essentially no FIP fractionation of low-FIP elements in the fast solar wind from polar coronal holes- the solar wind abundances are equal to the photospheric abundances within the uncertainties (Gloeckler \& Geiss 2007). This is an indication that the flow in the upper chromosphere is indeed fast.

One might expect that as one moves deeper into the chromosphere, where densities, and thus the collisional coupling, are much stronger, eventually all species must flow at the $\mathrm{H}$ velocity. Figure 4 shows that this is not correct; the relative flow speed difference between minor elements and $\mathrm{H}$ does not get smaller as the chromospheric density increases. The reason is simply that the frictional drag is not proportional to the $\mathrm{H}$ density but rather to the flux density $F_{\mathrm{H}}$, which remains constant throughout the chromosphere. Hence, one must expect that element fractionation will occur throughout a steady-state chromosphere. In a non-steady chromosphere, subject to, e.g., turbulent mixing, this is no longer the case: the fractionation takes place much more slowly in the dense lower chromosphere, and hence even very infrequent mixing events are sufficient to maintain fairly constant elemental abundances here, while the mixing must take place much more frequently in the upper chromosphere to offset the rapid gravitational settling there.

Helium, the most abundant element except hydrogen, has not been included in this study. It is a high-FIP element like neon, and since it is lighter it will put a less stringent requirement than neon on the funnel expansion factor. However, helium is not a minor element and may affect the flow of hydrogen as well as minor element flow in the chromosphere. The hydrogen flux densities assumed in our models are dictated by observations. In model calculations (e.g., Bürgi \& Geiss 1986; Hansteen et al. 1997; Lie-Svendsen et al. 2003), the same values of the $\mathrm{H}$ flux density can be produced with or without helium included. Since it is the $\mathrm{H}$ flux density that affects the minor element flow, and the $\mathrm{H}$ flux density is an input parameter to the model, the presence of helium will have little impact on the frictional coupling between hydrogen and the minor elements. The interesting issue is, however, the direct effect of helium on the heavier elements caused by the additional drag. If helium itself undergoes gravitational settling (small $f_{\max }$ ) it will also quickly become a minor element in the chromosphere, and its effect on other elements can be neglected. Hence, helium becomes important when $f_{\max }$ is sufficiently large to prevent settling of helium. As with the heavier elements, we expect from Equation (7) that settling becomes important when $F_{\mathrm{H}} \lesssim g / \hat{v}$. Choosing $\hat{v}=3 \times 10^{-16} \mathrm{~m}^{3} \mathrm{~s}^{-1}$ for $\mathrm{He}-\mathrm{H}$ collisions (Banks \& Kockarts 1973), we find $g / \hat{v} \approx 9 \times 10^{17} \mathrm{~m}^{-2} \mathrm{~s}^{-1}$, which is less than half of even the smallest $F_{\mathrm{H}}$ that we have assumed (corresponding to $f_{\max }=22$ ). Thus, helium will not undergo gravitational settling in any of the three flow geometries that we have assumed. Let us therefore assume that in these models helium would flow at approximately the hydrogen velocity and with a constant abundance throughout the slab. Since in this case $f[\mathrm{He}] \approx 1$ we set the chromospheric He abundance equal to the observed fast solar wind abundance, $A_{\mathrm{He}}=0.05$ (von Steiger et al. 2000, implying that any fractionation has taken place below the lower boundary of the model). Since helium is assumed to flow at the hydrogen velocity with a constant abundance, we may replace $v_{i \mathrm{H}}$ (describing collisions between heavy elements and hydrogen) with an "effective" collision frequency $v^{\prime} \equiv v_{i \mathrm{H}}+v_{i \mathrm{He}}$. Furthermore, assuming hardsphere interactions and using that the cross section for collisions between neutral heavy elements and helium is approximately equal to the cross section for collisions with hydrogen, and 
that the heavy element is much heavier than both helium and hydrogen, we may make the replacement (see Schunk 1977)

$$
\hat{v} \rightarrow \hat{v}^{\prime}=\hat{v}\left(1+A_{\mathrm{He}} \sqrt{\frac{m_{\mathrm{He}}}{m_{p}}}\right)=1.1 \hat{v}
$$

in Equations (11) and (12). Since settling sets in when $F_{\mathrm{H}}<$ $g / \hat{v}$, the new, approximate, criterion for settling is then $F_{\mathrm{H}} \lesssim$ $0.9 g / \hat{v}$. Above we have argued that preventing settling of neon requires $f_{\max } \gtrsim 30-40$. If helium had been included, we thus estimate that this threshold for $f_{\max }$ would be lowered by approximately $10 \%$. Thus, we may conclude that the inclusion of helium should not affect the main results obtained here, although its impact may still be sufficiently large to warrant another study in which the coupled hydrogen and helium transport equations are solved simultaneously (as was done in the models of Hansteen et al. 1997 and Lie-Svendsen et al. 2003).

Although helium has little direct impact on the chromospheric flow, it can modify the chromosphere indirectly through its impact in the corona. Funnel models of the solar wind, with helium included and not treated as a minor species, have shown that the helium abundance in the corona may become very large and thus have a large impact on the flow of hydrogen in the corona through Coulomb collisions between the two species, and helium may even trigger a transition from a high-speed wind with a low coronal helium abundance to a slow-speed wind with a high coronal helium abundance (Janse et al. 2007). Since the hydrogen flux, $F_{\mathrm{H}}$, is determined by the coronal energy balance, helium therefore cannot be neglected in solar wind models that aim to compute $F_{\mathrm{H}}$. Again, in the simple model used here, where $F_{\mathrm{H}}$ is an input parameter to the model, this is not an issue.

We have assumed that some, unspecified, mixing process maintains photospheric abundances up to the lower boundary of the model. If no such mixing were to occur at all in the solar atmosphere, this treatment would of course be incorrect; settling would then occur throughout the chromosphere, and there would be no justification for assuming photospheric abundances at some arbitrarily chosen lower boundary since abundances at this boundary would also be affected by the settling. However, we do know that convective mixing indeed occurs at least in the photosphere and up toward the temperature minimum (above which the atmosphere is convectively stable). And since even infrequent mixing events are sufficient to offset gravitational settling in the lower chromosphere, it seems reasonable to assume that little fractionation will occur there. However, assuming that the chromosphere remains well mixed up to $1 \mathrm{Mm}$ below the transition region, in the mid-chromosphere, as we do in our numerical model, is certainly an arbitrary assumption. The main results that we have obtained are not very sensitive to the placement of the lower boundary, however. When the funnel is sufficiently narrow so that $F_{\mathrm{H}}>g / \hat{v}$, Equation (12) shows that the (maximum) fractionation is independent of where we put the lower boundary. Conversely, when $F_{\mathrm{H}}<g / \hat{v}$, the fractionation is proportional to $\exp (-\gamma \tau)$ (Equation (11)) and thus extremely sensitive to the slab thickness $\tau$. However, in this case, because $\gamma \gg 1$, the main result is simply that no particles are pulled out of the chromosphere. For instance, with $F_{\mathrm{H}}=2 \times 10^{18} \mathrm{~m}^{-2} \mathrm{~s}^{-1}\left(f_{\max }=22\right)$ and $\tau=4$ (close to the actual number of $\mathrm{H}$ scale heights in the model), Equation (11) predicts $f[\mathrm{Ne}] \approx 5 \times 10^{-13}$, while with $\tau=0.4$ we find $f[\mathrm{Ne}] \approx 0.04$. Only with $\tau \ll 1$, corresponding to mixing taking place throughout the chromosphere up to only a few $\mathrm{Ne}$ scale heights (which is only of order $10 \mathrm{~km}$ ) below the transition region, would the neon fractionation be of order unity. In other words, when settling occurs $\left(F_{\mathrm{H}}<g / \hat{v}\right)$ the solar wind abundances drop by many orders of magnitude nearly irrespective of where we put the lower boundary of our model (equivalent to the upper boundary of the mixing).

Flow in narrow funnels may explain why solar wind abundances do not deviate by orders of magnitude from photospheric abundances. However, if the wind originates in such funnels, it implies that most of the chromosphere underlies magnetically closed regions, where there can be no net outward flow. Maintaining fairly constant abundances throughout a chromosphere without net hydrogen outflow would require steady mixing on a fairly short timescale (minutes). Since the hydrogen density and temperature structure should be quite similar with or without flow, it is difficult to argue that the chromosphere outside the funnels should be subject to more mixing than inside the funnels. If our picture of an upper chromosphere subject to gravitational settling is correct, this region should be more or less depleted of heavy, high-FIP elements in magnetically closed regions of the Sun. However, this does not mean that the overlying coronal loops would be depleted as well: as shown by Killie \& Lie-Svendsen (2007), for minor ions the transition region acts like a barrier between the chromosphere and corona. Thermal forces in the transition region are so strong that minor ions, even heavy ions like iron, are unable to flow from the corona to the transition region unless there is a strong downflow of hydrogen as well (which requires a large reduction in loop heating). The coronal abundance is therefore maintained even as the abundance in the upper chromosphere is severely depleted by gravitational settling; "ordinary" diffusion (given by the pressure gradient force) is simply not able to counteract the thermal diffusion unless the abundance varies by orders of magnitude across the transition region. A possible scenario is then that the elemental composition of coronal loops is determined when they are formed, either through flux emergence or by initial filling of a nearly "empty" loop, in which case the rapid hydrogen flow can prevent the chromospheric gravitational settling, but thereafter the loop composition is decoupled from the chromospheric composition. For that reason, the fact that loop abundances comparable to photospheric abundances are indeed observed (Raymond et al. 1997) is not incompatible with an upper chromosphere depleted of heavy elements.

The thermal forces that operate in the transition region provide another argument in favor of gravitational settling taking place in the upper chromosphere: if a well-mixed chromosphere is maintained all the way up to the transition region interface, high-FIP elements (which are neutral in the region where hydrogen is ionized) are pulled from the top of the chromosphere into the coronal loop by thermal diffusion, while low-FIP elements (which are ionized before hydrogen) cannot flow through the hydrogen ionization layer (Killie et al. 2005; Killie \& Lie-Svendsen 2007). As a result an inverse FIPeffect would result, with the loop becoming overabundant in high-FIP elements and depleted in low-FIP elements. Since observations show the opposite effect in quiescent loops of our Sun, this indicates that the chromosphere cannot remain wellmixed throughout.

We have treated the solar wind outflow from funnels as a one-dimensional problem, although the magnetic field structure in the chromosphere and low corona is expected to be far from one dimensional (e.g., Dowdy et al. 1986). For our purpose that three-dimensional topology is irrelevant, as we are only interested in the flow along one open funnel, and whether the 
flow is sufficiently fast to pull heavy elements out from the chromosphere. However, if the funnel is sufficiently narrow the "leakage" from the funnel to the adjacent closed loops may become appreciable, and the problem must be treated as a twodimensional problem at least. Neutral particles that are not subject to rapid charge-exchange reactions can easily diffuse across field lines. At chromospheric densities with a funnel magnetic field strength of order $0.01 \mathrm{~T}$ (Dowdy et al. 1986), the charged particle diffusion speed across field lines will be less than $10^{-5}$ times the neutral particle diffusion speed, and can be neglected.

For the hydrogen background the leakage is negligible: since hydrogen is close to hydrostatic equilibrium whether it is flowing or not (as long as the flow is subsonic), the hydrogen pressure difference between the funnel and adjacent closed loops at the same altitude in the chromosphere must be small. The only difference will be in the ionization degree; as we have seen, the rapid funnel flow leads to a much lower ionization degree, and there will thus be a small flow of neutral hydrogen from the funnel to the adjacent loops. However, this loss of neutral hydrogen will be very small because of the rapid charge exchange between protons and neutral hydrogen, which essentially forces them to flow together. Secondly, we have already seen that our results do not depend sensitively on the hydrogen ionization degree (Section 3.4), so that a small change in the chromospheric ionization resulting from the leakage will have no impact on our results.

We are then left with the possible effect of leakage of neutral, minor elements from the funnel. Since neutral oxygen is constrained to follow field lines because it is tightly coupled to ionized oxygen through the resonant charge exchange with protons, and iron is fully ionized in the funnel and therefore cannot diffuse across field lines, we are left considering neon. With the largest expansion factors considered, $f_{\max }=30-40$, the neon scale height in the funnel is comparable to the hydrogen scale height. Assuming that there is no outflow in the neighboring loops, neon is assumed to have settled gravitationally there, so the density in the upper chromosphere is essentially zero, compared to the funnel at the same altitude. The resulting pressure difference will set up a flow of neon from the funnel to the loops, resulting in a solar wind neon flux (and hence fractionation) that is lower than we obtained in our one-dimensional model. In order to estimate this loss, assume that the funnel is a cylinder with radius $h$ and height $\Delta z$, and that the neon density is zero outside the funnel. The horizontal pressure gradient will then be of order $d P / d x \sim n k T / h$ where $n$ is the neon density inside the funnel. This pressure gradient is balanced by friction with hydrogen, resulting in a horizontal diffusion speed of order $u_{D}=k T /\left(m h \hat{v} n_{\mathrm{H}}\right)$. Assuming the vertical flow is so rapid that the neon abundance, $A_{\mathrm{Ne}}$, does not vary with altitude, we estimate a horizontal diffusion flux density $F_{D} \sim A_{\mathrm{Ne}} n_{\mathrm{H}} u_{D}=A_{\mathrm{Ne}} k T /(m h \hat{v})$, which is only weakly dependent on altitude. We then define the loss, or leakage, from the funnel, $L$, as the ratio of the integrated leakage flux to the solar wind flux through the top surface of the cylinder,

$$
L=\frac{2 \pi h \Delta z A_{\mathrm{Ne}} \frac{k T}{m h \hat{v}}}{\pi h^{2} A_{\mathrm{Ne}} F_{\mathrm{H}}}=2 \frac{\Delta z}{h} \frac{k T}{m \hat{v} h F_{\mathrm{H}}} .
$$

Using Dowdy et al.'s estimate for the size of these structures, $h \sim 1 \mathrm{Mm}$, our slab thickness $\Delta z=1 \mathrm{Mm}, T=8 \times 10^{3} \mathrm{~K}$, $\hat{v}=8 \times 10^{-17} \mathrm{~m}^{3} \mathrm{~s}^{-1}$ (corresponding to hard-sphere collisions between neutral $\mathrm{Ne}$ and neutral $\mathrm{H}$ ), and our largest funnel expansion with $F_{\mathrm{H}}=4 \times 10^{18} \mathrm{~m}^{-2} \mathrm{~s}^{-1}$, we find

$$
L \approx 0.02 \text {. }
$$

Since variations in fluxes at the percent level are irrelevant for this study (as we have seen, fluxes can vary by orders of magnitude when the funnel expansion is changed), this leakage can be neglected and the one-dimensional treatment should be more than adequate for our purpose. However, if improved observational resolution were to show that funnels are much narrower than this, of order $100 \mathrm{~km}$ or less in horizontal extent, it may be necessary to extend the calculation to two (or three) dimensions for the high-FIP elements, in order to account for this loss.

\section{CONCLUSION}

We have shown that if the fast solar wind originates in chromospheric funnels, the hydrogen flow in the funnel can be sufficiently fast so that the frictional drag from hydrogen can prevent gravitational settling of heavier elements and lead to solar wind abundances comparable to photospheric abundances, provided sufficient minor ion heating in the corona. This frictional drag is sensitive to the charge state of the heavy element, because of the magnitude of the Coulomb cross section for collisions between ions and protons. Consequently, iron, a low-FIP element which is fully ionized in the upper chromosphere, does not undergo gravitational settling for the chosen funnel expansion factors $f_{\text {max }}$. Neon, a mostly neutral high-FIP element, undergoes extensive gravitational settling despite that it has less than half of the atomic mass of iron. Oxygen, a high-FIP element, is an exception. Because of the resonant charge exchange reaction between oxygen and hydrogen, which dominates the force balance, oxygen does not undergo extensive settling even when the oxygen ionization degree is low. A small degree of hydrogen ionization is sufficient to prevent settling of low-FIP elements.

The solar wind abundances can be extremely sensitive to the value for the funnel expansion factor $f_{\text {max }}$; a small reduction in $f_{\max }$ can trigger a transition from an abundance comparable to the photospheric abundance to an abundance many orders of magnitude less.

Of the three minor elements considered, $\mathrm{O}, \mathrm{Ne}$, and $\mathrm{Fe}$, neon puts the most stringent requirement on $f_{\max }$. The calculations presented here show that $f_{\max } \gtrsim 30-40$ is needed for the solar wind neon abundance to be comparable to the photospheric abundance. Since neon does not have suitable photospheric lines, there is no direct information on the photospheric abundance. It is therefore possible that neon does undergo significant settling, with the observed solar wind abundance being much smaller than the (unobserved) photospheric abundance. However, from helioseismology it is estimated that the absolute neon abundance of the Sun could be about $(2-3) \times 10^{-4}$ (Antia \& Basu 2005; Bahcall et al. 2005). Assuming that the solar wind oxygen abundance is equal to the photospheric abundance given by Asplund et al. (2005), and using a solar wind neon abundance relative to oxygen of 0.07 (Gloeckler $\&$ Geiss 2007), the absolute solar wind neon abundance is $A_{\mathrm{Ne}}^{\mathrm{sw}} \approx 3 \times 10^{-5}$. Assuming that the solar abundance inferred from helioseismology is equal to the photospheric abundance, we thus obtain from observations an absolute neon fractionation $f[\mathrm{Ne}] \approx 0.1$ (which is of course also the fractionation relative to oxygen with these assumptions). We have shown that once gravitational settling sets in, the solar wind abundance may quickly 
drop by much more than a factor of 10; e.g., Table 2 shows the maximum fractionation $f[\mathrm{Ne}] \sim 10^{-3}$ for $f_{\max }=32$. In the absence of direct measurements of the photospheric neon abundance, helioseismology and in situ solar wind observations thus still seem to require a funnel expansion $f_{\max } \gtrsim 30-40$.

Taking the large-scale coronal hole expansion into account (which may take place over a solar radius or so), assuming that this alone increases the flow tube area by roughly a factor of 7 (Munro \& Jackson 1977), the actual funnel expansion taking place somewhere in the low corona must increase the flow tube area by approximately a factor of 5 or more. Based on measured blueshifts of a coronal Ne VIII line, and the absence of blueshifts in transition region C and O lines (e.g., Peter \& Judge 1999), Byhring et al. (2008) found that this latter expansion factor could not be much more than 4 . Given the uncertainties, it therefore seems that such a modest funnel expansion, which is much smaller than the expansion originally suggested by Dowdy et al. (1986), is consistent with measurements of both in situ solar wind abundances and Doppler shifts in transition region and coronal lines.

\section{REFERENCES}

Antia, H. M., \& Basu, S. 2005, ApJ, 620, L129

Arnaud, M., \& Raymond, J. 1992, ApJ, 398, 394

Arnaud, M., \& Rothenflug, R. 1985, A\&AS, 60, 425

Asplund, M., Grevesse, N., \& Sauval, A. J. 2005, in ASP Conf. Ser. 336, Cosmic Abundances as Records of Stellar Evolution and Nucleosynthesis, ed. T. G. Barnes III \& F. N. Bash (San Francisco, CA: ASP), 25
Bahcall, J. N., Basu, S., \& Serenelli, A. M. 2005, ApJ, 631, 1281 Banks, P. M., \& Kockarts, G. 1973, Aeronomy (New York: Academic) Bürgi, A., \& Geiss, J. 1986, Sol. Phys., 103, 347

Byhring, H. S., Esser, R., \& Lie-Svendsen, Ø. 2008, ApJ, 673, L91

Cranmer, S. R., Panasyuk, A. V., \& Kohl, J. L. 2008, ApJ, 678, 1480

Dowdy, J. F., Jr., Rabin, D., \& Moore, R. L. 1986, Sol. Phys., 105, 35

Gloeckler, G., \& Geiss, J. 2007, Space Sci. Rev., 130, 139

Hansteen, V. H., Leer, E., \& Holzer, T. E. 1997, ApJ, 482, 498

He, J.-S., Tu, C.-Y., \& Marsch, E. 2008, Sol. Phys., 250, 147

Janse, A. M., Lie-Svendsen, Ø., \& Leer, E. 2007, A\&A, 474, 997

Killie, M. A., \& Lie-Svendsen, Ø. 2007, ApJ, 666, 501

Killie, M. A., Lie-Svendsen, Ø., \& Leer, E. 2005, ApJ, 632, L155

Korevaar, P., \& van Leer, B. 1988, A\&A, 200, 153

Landau, L. D., \& Lifshitz, E. M. 1999, Course on Theoretical Physics, Vol. 6, Fluid Mechanics (2nd ed.; Oxford: Butterworth-Heinemann)

Lie-Svendsen, Ø., \& Esser, R. 2005, ApJ, 618, 1057

Lie-Svendsen, Ø., Hansteen, V. H., \& Leer, E. 2003, ApJ, 596, 621

Mazzotta, P., Mazzitelli, G., Colafrancesco, S., \& Vittorio, N. 1998, A\&AS, 133,403

McKenzie, J. F. 2000, Sol. Phys., 196, 329

McKenzie, J. F., Sukhorukova, G. V., \& Axford, W. I. 1998, A\&A, 332, 367

Munro, R. H., \& Jackson, B. V. 1977, ApJ, 213, 874

Péquignot, D., Petitjean, P., \& Boisson, C. 1991, A\&A, 251, 680

Peter, H. 1998, A\&A, 335, 691

Peter, H., \& Judge, P. G. 1999, ApJ, 522, 1148

Raymond, J. C., et al. 1997, Sol. Phys., 175, 645

Schultz, D. R., Krstic, P. S., Lee, T. G., \& Raymond, J. C. 2008, ApJ, 678, 950

Schunk, R. W. 1977, Rev. Geophys. Space Phys., 15, 429

Shull, J. M., \& van Steenberg, M. 1982, ApJS, 48, 95

Vernazza, J. E., Avrett, E. H., \& Loeser, R. 1981, ApJS, 45, 635

Verner, D. A., \& Ferland, G. J. 1996, ApJS, 103, 467

von Steiger, R., Geiss, J., Gloeckler, G., \& Galvin, A. B. 1995, Space Sci. Rev., 72,71

von Steiger, R., et al. 2000, J. Geophys. Res., 105, 27217 